\title{
DESENVOLVIMENTO DE COLEÇÕES EM COLEÇÕES DIGITAIS
}

\section{COLLECTION DEVELOPMENT IN DIGITAL COLLECTIONS}

${ }^{1}$ Anastácia Freitas de Oliveira

${ }^{2}$ Murilo Bastos da Cunha

Universidade de Brasília ${ }^{1,2}$

\section{Correspondência}

Anastácia Freitas de Oliveira

Universidade de Brasilia

Brasília, DF - Brasil

E-mail: anastacia.oliveira1@gmail.com

(iD) http://orcid.org/0000-0002-0139-1977

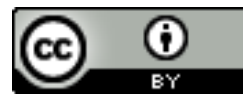

JITA: HO. e-books.

e-Location: 019025 


\section{RESUMO}

Relata as mudanças de paradigmas ocorridas na transição de coleções físicas para as coleções digitais. Apresenta as recomendações para seleção, criação e manutenção dessas coleções, a partir do exame de literatura especializada, abordando aspectos do conteúdo, viabilidade técnica, funcionalidade, confiabilidade, suporte do fornecedor, licenciamento, preservação, acessibilidade e questões contratuais. Discorre sobre aspectos que devem ser observados na tomada de decisão sobre a escolha de manter ou não documentos impressos quando há o equivalente digital. Conclui com a síntese das recomendações, indicando também estudos relacionados ao desenvolvimento de coleções.

\section{PALAVRAS-CHAVE}

Desenvolvimento de coleções. Documento eletrônico. Bases de dados.

\section{ABSTRACT}

Reports paradigm changes that occurred in the transition from physical collections to digital ones. It presents recommendations for creation and maintenance of these collections, based on the examination of specialized literature, addressing aspects of content, technical feasibility, functionality, reliability, supplier support, licensing, preservation, accessibility and contractual issues. It discusses aspects that must be observed in the decisionmaking on the choice to keep or not printed documents when the digital equivalent exists. It concludes with the synthesis of the recommendations, also indicating studies related to the collection development.

\section{KEYWORDS}

Collections development. E-Book. Databases. 


\section{Introdução}

O acesso a documentos por meio da disponibilização em meio digital já faz parte de diversos tipos de bibliotecas há vários anos. Seja em função da aquisição de bases de dados, seja por meio de compra de livros eletrônicos, vários gestores optam por oferecer coleções digitais por diversas vantagens: diversificação do conteúdo, acesso simultâneo e independente de localização geográfica, diminuição dos custos de compra e armazenamento, redução de tempo de processamento técnico dos documentos, dentre outros motivos.

Buckland (1995) arrola outros benefícios muito importantes, como a expansão do acesso e a possibilidade de "customização" da coleção por uma conveniência geográfica, uma vez que diversos grupos de interesse podem ser atendidos por meio da cooperação entre instituições e o acesso simultâneo a itens.

Nesta realidade, será necessário avaliar aspectos próprios desse novo contexto onde o suporte físico e eletrônico convivem, como o custo de acesso e a possibilidade de cooperação bibliotecária. Na visão de Vergueiro (2000), a importância social da atividade de seleção tem sido incrementada, ao invés de minimizada, pelas tecnologias de informação eletrônica. Romero (2007) acrescenta que o advento dos suportes digitais tornou o processo de seleção, aquisição e organização de documentos mais complicado do que nunca.

Uma das mudanças de paradigma ocorridas com o advento das coleções eletrônicas é, segundo Dias e Pires (2003), que o valor da biblioteca do futuro não será medido por tamanho, idade e abrangência das coleções, mas sim avaliadas pela força de acessibilidade às interconexões com rede.

Mais uma modificação salientada por Gregory (c2011) é que os bibliotecários muitas vezes selecionam um material disponível na web, mas não o colecionam de fato. A depender da licença do material é possível apenas direcionar o usuário a uma página e não possuir o conteúdo. Já no fim dos anos 1980, Buckland (1989) argumentava que o crescimento do acesso remoto a materiais faria com que a posse seja menos importante se comparada com o acesso.

Outra mudança substancial é citada por Cunha (2009), que apontava que, diferentemente do passado, os recursos informacionais podem ter diversas origens: "materiais originalmente digitais, periódicos eletrônicos com licença de uso, livro eletrônico e materiais substitutos de obras impressas".

Horava (2010) menciona o receito de muitos bibliotecários em relação aos documentos eletrônicos. Entretanto, o autor afirma:

Nossas coleções não estão desaparecendo. Pelo contrário, eles estão se tornando extraordinariamente importantes à medida que nossa presença na Web nos permite novas capacidades para nos conectarmos e sermos relevantes para a população que 
servimos. Ao focar no acesso remoto, gestão ativa (localmente e em colaboração com grupos externos), uma maior conscientização sobre os resultados de aprendizagem e a agenda do pesquisador, e uma abordagem sustentável com nuances cuidadosas para a atividade de aquisição, podemos aumentar o valor e uso de nossas coleções.

Apesar do receio de alguns profissionais e da mudança de paradigmas, Schmidt (2004) avalia que a transformação ocorrida no processo de seleção tratava-se de um passo natural adiante, mesmo que aparentemente entre em conflito com os valores e história já arraigados.

O exame da literatura especializada do tema pode auxiliar os profissionais da informação no desenvolvimento de coleções digitais sustentáveis, inteligentes e que atendam adequadamente às demandas informacionais dos usuários. Por isso, o objetivo desse artigo é identificar aspectos que devem ser observados para a criação e manutenção de coleções digitais. Para alcançar esse objetivo, a metodologia utilizada será a revisão de literatura, por meio de exame seletivo dos documentos sobre o tema, dando ênfase aos preceitos de associações e organizações que se debruçaram sobre o assunto. A revisão considera a transição de parte de preceitos conceituais e das características intrínsecas aos documentos eletrônicos.

\section{Revisão de Literatura}

A revisão de literatura deste trabalho foi narrativa. Conforme Cordeiro et. al. (2007, p. 429-430) na revisão da literatura narrativa ou tradicional a busca das fontes não é prédeterminada e específica, sendo frequentemente menos abrangente. A seleção dos artigos tem interferência da percepção subjetiva, portanto, é arbitrária.

O primeiro passo foi o exame das teses e dissertações mais recentes sobre "desenvolvimento de coleções" aplicado ao contexto digital. A partir da observação dos termos e autores mais recorrentes, partiu-se para a busca nos periódicos científicos nacionais e internacionais.

A pesquisa bibliográfica em suportes eletrônicos utilizou as bases de dados disponíveis pelo Portal de Periódicos Capes, principalmente as bases Library and Information Science Abstracts - LISA; Library, Information Science \& Technology Abstracts with Full Text LISTA e EMERALD. O sumário de periódicos específicos como Library collections: acquisitions \& technical services e Collection building também foram examinados a fim de localizar a produção bibliográfica específica sobre o tema.

Destaca-se a busca de materiais dentre os documentos da International Federation of Library Associations and Institutions - IFLA e da National Information Standards Organization - NISO onde foram encontradas publicações com recomendações para a construção de coleções digitais. 
Em âmbito nacional, foram utilizadas as bases de dados Arquivologia, Biblioteconomia, Ciência da Informação, Documentação e Museologia - ABCDM; Bases de Dados em Ciência da Informação - BRAPCI e a Biblioteca Digital Brasileira de Teses e Dissertações - BDTD.

As palavras-chave utilizadas nas bases de dados nacionais foram "biblioteca(s) digital(is) E desenvolvimento de coleção(ões)"; "desenvolvimento de coleção(ões)"; "gestão de acervo(s)" e "livro(s) eletrônico(s)".

Em seguida, utilizaram-se as seguintes palavras-chave para recuperar a literatura científica internacional: "collection development"; "collection management"; "e-collection"; "ebooks"; "collection development policies".

Quando necessário, realizou-se uma consulta específica para esclarecer conceitos técnicos, como nos tópicos de usabilidade e acessibilidade.

\subsection{Conteúdo}

Em geral, os critérios de avaliação de conteúdo de documentos analógicos podem ser utilizados para o exame de documentos eletrônicos. No entanto, uma série de critérios de conteúdo exclusivo para recursos eletrônicos devem ser considerados, sobretudo para determinar a opção entre o formato físico e o digital.

Johnson et. al. (2012, p. 6) elaboraram o guia "Key issues for e-resource collection development: a guide for libraries", uma publicação institucional da IFLA. No documento, os autores afirmam que os critérios devem apoiar os principais objetivos da organização; complementar ou adicionar profundidade e amplitude à coleção existente com base em perfis de assuntos; ser de certa qualidade (por exemplo, revisto por pares, ou ter um produtor reputado); dar suporte às necessidades do público-alvo e gerar um nível aceitável de utilização.

Rodrigues e Carvalho (2003, p. 7) reforçam que os aspectos relativos ao conteúdo dos documentos digitais são muito semelhantes aos dos documentos impressos: o âmbito e a abrangência temática, a autoridade dos autores ou produtores do recurso, a atualização, a organização e a apresentação da informação. No caso de versões digitais de documentos que existem também em suporte impresso, os autores pedem especial atenção para o valor acrescentado da versão digital (por exemplo, outros tipos de conteúdo ou funcionalidades de utilização).

Já Johnson et. al. (2012, p. 6) acrescentam a consistência da publicação eletrônica face ao equivalente impresso, a disponibilidade de edições anteriores, a atualidade do conteúdo online e frequência de atualizações como critérios em relação ao conteúdo que dispensam especial atenção. 
Por sua vez, Gregory (c2011, p. 62) alerta que determinar a acurácia de materiais disponíveis em meio eletrônico, sobretudo na World Wide Web é fator crítico no processo de seleção e avaliação. Materiais gratuitos devem receber especial atenção, sobretudo se não forem produzidos por autores ou entidades renomadas.

\subsection{Viabilidade técnica}

São aspectos de viabilidade técnica de acordo com Johnson et. al. (2012):

- Disponibilidade: acesso remoto e acesso individual. O acesso remoto via Web é muitas vezes preferível, porque proporciona benefícios adicionais como a atualização mais rápida, o acesso otimizado, a redução de encargos em termos de armazenamento e redução de custos com a conservação e manutenção;

- Autenticação: filtragem de senha ou de acesso via Internet Protocol - IP. Normalmente permite-se o acesso simultâneo. Também é possível o acesso por meio de um servidor proxy; dessa forma os usuários podem acessar os conteúdos eletrônicos fora dos limites físicos da biblioteca. $\mathrm{O}$ acesso pelo uso de senha não é recomendado, pois pode trazer dificuldades de controle de acessos, já que um membro cadastrado pode, indevidamente, repassar a senha para uma pessoa não autorizada;

- Compatibilidade de hardware e software: A princípio, o recurso deve ser compatível com os dispositivos já existentes. No entanto, caso seja necessário, deve ser dada atenção ao custo adicional de aquisição, instalação e suporte de componentes de software ou de multimídia apropriados. Outro fator de compatibilidade é o navegador, que deve ser ajustado de acordo com os requisitos nos computadores locais. A determinação dos formatos de arquivo (HTML, SGML, XML, PDF, epub, etc.; e formatos como JPEG, MPEG, etc.) que as bases de dados usam é importante: a depender do propósito, tamanho e tipo de documento será desejável um tipo de arquivo específico.

\subsection{Funcionalidade e Confiabilidade}

Considerado uma funcionalidade essencial, o mecanismo de busca deve ser poderoso, flexível e fácil de usar. De acordo com Johnson et. al. (2012, p. 8) as características habituais incluem a pesquisa por palavras-chave e pesquisa booleana, pesquisa de texto integral, possibilidade de percorrer índices e títulos, ordenação por relevância, tesauro, truncamento, navegação, histórico de pesquisa e transliteração.

Os autores acrescentam (2002, p. 9) que a exportação e a transferência de arquivos é requisito desejável. A impressão, envio por correio eletrônico, download para o computador,

\begin{tabular}{l|l|l|l|l|l}
\hline (c) RDBCl: Rev. Digit. Bibliotecon. Cienc. Inf. & Campinas, SP & v.17 & $1-20$ & e019025 & 2019 \\
\hline
\end{tabular}


para outro dispositivo eletrônico ou para software de gestão bibliográfica (como Endnote, Mendeley, BibTex, etc.) são vantagens a serem consideradas. A impressão deve ser fácil e o download não deve ter quaisquer restrições ou taxas adicionais impostas.

Algumas ferramentas também podem ser utilizadas concomitantemente nas coleções digitais, permitindo a integração dos fluxos de trabalhos e usuários finais. A NISO, em documento intitulado "A Framework of Guidance for Building Good Digital Collections" cita como exemplos dessa integração a folksonomia ou "social tagging" (2007, p. 23).

Johnson et. al. (2012, p. 9) apresentam a necessidade de o sistema suportar a integração com outros recursos através da ligação de referência e texto completo. O conteúdo deverá ser indexado em ferramentas de descoberta para facilitar a pesquisa e acesso eficaz a recursos locais e remotos.

A NISO (2007, p. 22) também reforça a importância da interoperabilidade. De acordo com a organização, uma boa coleção é interoperável, ou seja, seus metadados devem ser encontrados por mecanismos de busca externos. Essa condição deve estar prevista contratualmente, incluindo as condições de disponibilização e qualidade desses metadados. Essa é uma das formas de expansão do uso das coleções e podem ajudar na sustentabilidade das mesmas.

A base de dados ou a biblioteca digital deve ser confiável e disponível. Isso significa acesso 24 horas por dia, sete dias por semana. Johnson et. al. (2012) sinalizam que o sistema deve ser estável, com evidentes restrições a interrupções não programadas. Também deve ser tecnologicamente atualizado e ter a capacidade e infraestrutura de rede adequada para suportar múltiplos utilizadores e tempos de resposta ótimos.

Além disso, o objeto deve ter autenticidade. A NISO (2007, p. 55) define autenticação como o ato de determinar se o objeto está de acordo com sua origem, estrutura e histórico documentados e se o objeto não foi corrompido ou alterado de maneira não autorizada.

\subsection{Suporte do fornecedor}

Conforme orientado por Johnson et. al. (2012, p. 10) o fornecedor deve oferecer:

- Treinamento de usuários e suporte: opção de formação inicial e contínua, incluindo o fornecimento de documentação ou manuais online.

- Disponibilização de versão de teste e demonstração de produtos: os períodos de teste (trials) são particularmente úteis no apoio ao processo de avaliação de um produto em termos das questões técnicas, funcionalidade e fiabilidade. 
- Suporte técnico e processo de notificação do sistema: gerir e comunicar com antecedência e de forma eficaz o tempo de inatividade planejado e mudanças de conteúdo ou plataformas.

- É desejável a "customização" com a identificação da biblioteca ou instituição. Pode ser utilizado o logotipo ou marca para esse fim.

- Relatórios estatísticos: auxiliam na compreensão do custo versus benefício dos produtos. Os dados preferencialmente devem estar de acordo com padrões reconhecidos, como Counting Online Usage of Networked E-resources COUNTER e/ou o Standard Usage Statistics Harvesting Initiative - SUSHI.

O COUNTER propõe um código de práticas e de protocolos que permite que editores e fornecedores relatem o uso de seus recursos de maneira consistente. Além disso, exige que os editores submetam as estatísticas de uso a uma auditoria independente e rigorosa. Dessa forma, os bibliotecários podem ter um melhor entendimento de como os conteúdos contratados são utilizados, conseguindo comparar estatísticas de uso de diferentes fornecedores e obter métricas úteis como custo por uso.

A versão 5 (Cohen, 2019) contém diversos indicadores de uso como: total de vezes que um item foi acessado, quantidade de capítulos ou artigos procurados e baixados por usuário, quantidade de vezes que um conteúdo completo foi baixado, total de buscas em uma base de dados, quantos usuários tiveram acesso negado em função da não contratação de um conteúdo, etc. Já o padrão SUSHI (ANSI/NISO Z39.93-2014) define o modelo de solicitação e resposta automatizada para a coleta de dados de uso de recursos eletrônicos (NISO, 2019).

\subsection{Licenciamento}

As questões legais envolvidas no uso e colecionamento de informações eletrônicas tornam-se cada vez mais complexas, sobretudo por conta dos novos formatos de recursos eletrônicos, equipamentos de escaneamento e softwares de reconhecimento ótico de caracteres (Optical Character Recognition - OCR). Gregory (c2011, p. 141-142) indica que, nesse contexto, softwares e hardwares de gerenciamento de direitos digitais (digital rights management - DRM) estão presentes em diversas questões do desenvolvimento de coleções.

Gregory (c2011) esclarece que os sistemas DRM incluem uma variedade de tecnologias que fornecem aos proprietários do direito uma variedade de níveis de controle sobre como os conteúdos digitais e serviços podem ser utilizados. Genericamente, as tecnologias DRM habilitam os titulares de direitos autorais a protegerem seus materiais disponíveis eletronicamente de um uso não autorizado por meio de hardware ou software e determina sob que circunstâncias os usuários podem acessar os conteúdos. Podem, por exemplo, habilitar ou restringir a impressão e o compartilhamento. Os componentes de um sistema DRM podem compreender em: contenções de segurança para impedir acessos não autorizados; manifestação de direitos; identificação e associação de metadados; identificação de pessoas e organizações

\begin{tabular}{l|l|l|l|l|l|}
\hline (C) RDBCl: Rev. Digit. Bibliotecon. Cienc. Inf. & Campinas, SP & v.17 & $1-20$ & e019025 & 2019 \\
\hline
\end{tabular}


com potencial de interação com o conteúdo; tecnologias para associar identificadores como marcas d'água e sistemas de pagamento.

Embora tenha diversas vantagens como a redução de custos de transação e a discriminação de preços, Bittar (2015, p. 65) alerta que o sistema DRM é constantemente questionado não tanto pela tecnologia em si, mas como ele é estruturado, pois ainda permite o uso para fins não relacionados com a proteção de direitos autorais. Ainda é preciso observar que o sistema em si não é infalível e não é capaz de impedir totalmente a pirataria.

$\mathrm{Na}$ tentativa de flexibilizar a relação entre os direitos de uso do autor e a necessidade do público de acessar a obra, a licença Creative Commons foi criada. Além de prover modelos de licença legíveis por humanos e máquinas com o valor legal reconhecido, a iniciativa esclarece de forma simples quais são as permissões e restrições para o público.

Há ainda os materiais não licenciados que podem ser de domínio público ou acesso aberto. O conteúdo é gratuito, geralmente não há a necessidade de autenticação e é possível fazer vários acessos simultâneos. Porém, Serra (2015, p. 146-148) aponta como desvantagem a baixa ou falta de credibilidade do material, eventual dificuldade de uso em função da qualidade; falta de manutenção e a ausência de garantia de persistência dos links para acesso em outras plataformas.

Além do controle de acesso, outro desafio é a definição de utilizadores e locais autorizados. Gregory (c2011, p. 149) alerta que o fornecedor define como "usuário" e "acesso" pode ser limitado a um computador ou prédio. Quem será responsável pelo uso não autorizado e quais serão as consequências de um eventual uso indevido?

\subsection{Preservação}

De acordo com Boeres (2009, p. 68) a informação digital deve estar preservada dentro dos limites e cuidados inerentes à tecnologia, buscando evitar que a informação fique indisponível com o tempo, por mau uso do documento, ou por obsolescência da mídia. Por esse motivo, a análise dos ônus inerentes a preservação digital dos conteúdos é passo fundamental.

Gregory (c2011, p. 196) esclarece que as iniciativas de preservação de materiais podem advir do próprio editor (ou mesmo do agregador ou vendedor), da biblioteca por si mesma ou de acordos cooperativos. Muitas vezes, o alto custo para iniciativas de preservação pela própria biblioteca desestimula essa prática, a exceção dos materiais produzidos de forma local. $\mathrm{O}$ arquivamento a longo prazo e a preservação de materiais digitais é um empreendimento difícil e caro que requer recursos substanciais e sério comprometimento. 
O arquivamento de dados é passo importante para a preservação. Johnson et. al. (2012, p. 10) advertem que a frequência de realização de backups e a oferta de dados em mídias físicas caso a empresa cesse ou transfira publicações deve ser considerado pelo responsável pela escolha determinados produtos. A biblioteca deve gerir o arquivamento e o acesso no formato oferecido, bem como as características que possam perder-se face ao recurso original. Ainda de acordo com os autores, deve-se levar em conta se há política de arquivamento do fornecedor dos recursos.

Existem diversas iniciativas que auxiliam na preservação digital de documentos como LOCKSS e Portico, mas há que se considerar na confiabilidade em delegar o arquivamento. É igualmente importante avaliar a cobrança de eventuais taxas ou restrições ao acesso; a previsão de migração de arquivos para novos formatos ou plataformas para acompanhar os avanços e o impacto do cancelamento ou rescisão do acesso perpétuo ao conteúdo previamente assinado.

A NISO (2007, p. 9) recomenda especial atenção a esses aspectos, que estão descritos como parte da curadoria digital dos dados. A organização também destaca a importância de estratégias de preservação que geralmente envolvem formato transformação, emulação de hardware/software, ou combinações dos dois.

Sobre a sustentabilidade das coleções, a NISO aconselha a persistência dos objetos (2007, p. 25). Um bom objeto existe em um formato suportado em plataformas atuais e plataformas futuras. A escolha de formatos de arquivo deve considerar não apenas o acesso, mas também sua eventual degradação. Por isso a qualidade do arquivo por si só deve ser considerada.

A Library of Congress recomenda o uso do PDF/A para a preservação a longo prazo. Os padrões PDF/A são desenvolvidos e mantidos por um grupo de trabalho com representantes do governo americano, indústria, academia e suporte ativo da Adobe Systems Incorporated. Além da própria Library of Congress, diversos repositórios acadêmicos e órgãos governamentais dos Estados Unidos recomendam o uso (LIBRARY OF CONGRESS, 2019). No Brasil o Conselho Superior da Justiça do Trabalho e diversos repositórios institucionais de Universidades Federais também adotam o PDF/A como formato de arquivo.

Outra iniciativa é identificação global do objeto. O uso de identificadores padronizados como o ISBN ou o DOI dificulta casos de ambiguidade entre os objetos, permitindo o mapeamento dependendo do contexto de uso. (NISO, 2007, p. 52).

\subsection{Acessibilidade}

De acordo com a NISO (2007, p. 12) a acessibilidade é a propriedade de ser utilizável por pessoas com deficiência. As interfaces devem ser projetadas para maximizar a usabilidade 
para pessoas com deficiências visuais, perda de audição, perda de mobilidade (por exemplo, dificuldade em usar um mouse) e até mesmo deficiências cognitivas.

A chegada das tecnologias de informação representou ganhos significativos para a comunidade que possui deficiências. Dando ênfase ao contexto da comunidade de pessoas com deficiência visual, Kavanagh e Sköld (2009, p. 56) salientam que o impacto das tecnologias interfere na possibilidade de flexibilizar como as bibliotecas produzem, armazenam e distribuem suas coleções. São exemplos a transformação da leitura por meio de áudio livros digitais ou mesmo a conversão de conteúdo utilizando-se um programa de tradução braile. A cópia braile impressa e displays/teclados portáteis são exemplos do uso aliado softwares e hardwares para benefício desse grupo de usuários.

Malheiros (2019, p. 126) salienta que os acervos digitais também representam um grande avanço na mobilidade, pois os usuários já não teriam que enfrentar as barreiras da acessibilidade física. Além disso, a autora explica que os acervos digitais podem ser alternativas mais econômicas, uma vez que um livro em braile custa três vezes mais que o livro comum, além das limitações de tiragem e espaço. Embora os avanços sejam inegáveis e substanciais, Malheiros (2019, p. 132) afirma que diversas diretrizes e padrões devem ser observados para que o conteúdo esteja de fato acessível a esses usuários. A autora cita os padrões do consórcio World Wide Web (W3C) e E-mag: modelo de acessibilidade do governo eletrônico como exemplos de instruções.

Em relação ao acervo, Malheiros (2019, p. 172) acrescenta que o acervo das bibliotecas digitais deve conter itens que se adaptem de forma a poderem ser lidos pelos programas de voz (recurso utilizado para usuários cegos) e em formato que possa ser ampliado (recurso utilizado por usuários com baixa visão). McNaught (2014, p. 35) recomenda também que exista a possibilidade de alterações de cor e contraste e textos alternativos para imagens e tabelas, assim programas de voz conseguem abranger todo o conteúdo. É de igual importância a compatibilidade com dispositivos de tecnologia assistiva.

O formato do arquivo também interfere na acessibilidade. McNaugth (2014, p. 35) explica que textos que são "fotografias" dos documentos e o sistema de flip podem dificultar ou impossibilitar o uso. Além disso, metadados usados para aparência tipográfica em vez de significado semântico podem eliminar benefícios para usuários com deficiências.

\subsection{Usabilidade}

Embora a qualidade do conteúdo das bibliotecas digitais ou base de dados seja o principal fator motivador para a escolha de um determinado produto, a usabilidade é essencial. A veiculação dos objetos digitais depende intrinsecamente do meio eletrônico, por isso a avaliação da usabilidade se torna tão importante. 
Mesmo capaz de atender a necessidade de informação de usuários, é possível que o público abdique o uso de determinado recurso em função da dificuldade de localizar e/ou utilizar as informações. Emery e Stone (2013, p. 11) afirmam, inclusive, que se um recurso não for tão fácil de usar como o Google, o usuário possivelmente irá procurar a informação em outro local.

Nielsen (2012) define usabilidade como um atributo de qualidade que avalia quão fácil são interfaces para o uso. $\mathrm{O}$ termo também se refere a métodos para facilitar o uso durante o processo de design.

Segundo o mesmo autor, usabilidade é definida por cinco componentes:

1. Aprendibilidade: facilidade para os usuários para realizar tarefas básicas na primeira vez que encontrar a interface;

2. Eficiência: rapidez com que podem realizar tarefas quando já interagiram com a interface;

3. Memorização: quando os usuários retornam ao sistema após um período em usá-lo, quão facilmente se pode restabelecer proficiência;

4. Erros: quantos erros os usuários cometem, quão grave são esses erros, e quão facilmente se podem recuperar;

5. Satisfação: Quão agradável é usar o design.

Ainda de acordo com Nielsen (2012) são atributos de qualidade de interfaces a:

1. Utilidade: a interface dispõe de características necessárias ao público final;

2. Usabilidade: interface de uso fácil e agradável;

3. Proveitoso: a interface é útil e tem usabilidade.

Johnson et. al. (2012, p. 8) citam como exemplos de funcionalidades de sistemas userfriendly os tutoriais online, os menus iniciais, as ajudas de navegação e opções de ajuda sensíveis ao contexto, as opções de personalização (como a subscrição de feeds / alertas de $e$ mail, possibilidade de guardar o histórico de pesquisa, etc.). A capacidade de classificação e ordenação de resultados (por exemplo: classificação por autor, título, data, relevância, conceitos, etc.) também pode ser citada como item presente em um sistema com usabilidade.

Existem diversos métodos para a avaliação da usabilidade de um sistema. Alguns exemplos são as listas de requisitos, heurística de sistemas, dentre outros testes que podem ser executados durante o desenho do sistema ou depois, quando o design já está consolidado.

Outro método é a avaliação centrada no usuário. De acordo com Bastien (2010) nessa metodologia os usuários são convidados a responder perguntas típicas sobre o produto, ou simplesmente são convidados a explorar a interface livremente enquanto os pesquisadores

\begin{tabular}{l|l|l|l|l|l}
\hline C RDBCl: Rev. Digit. Bibliotecon. Cienc. Inf. & Campinas, SP & v.17 & $1-20$ & e019025 & 2019 \\
\hline
\end{tabular}


observam e registram pontos que levam a erros ou causam dificuldades. Uma vez identificadas falhas no projeto, são propostas melhorias na qualidade da ergonomia ou mudanças no design.

\subsection{Aspectos contratuais}

Os responsáveis pela seleção e aquisição de bases de dados devem revisar cuidadosamente os modelos de negócio disponíveis no mercado. Segundo Arora (2018) não há modelo de precificação padrão para recursos eletrônicos, já que geralmente as propostas são baseadas em vários critérios e variáveis, como o tamanho da população de usuários e o número de usuários simultâneos.

Costa; Cunha (2005) apontam como modelos de negócio o acesso perpétuo, a assinatura, o empréstimo de curto prazo e a aquisição orientada pelo usuário. Cada modelo deve ser avaliado de acordo com o contexto da unidade de informação contratante, que deve considerar, dentre outros aspectos: o número de usuários e suas necessidades de informação, variedade e qualidade do conteúdo e, obviamente, orçamento disponível.

Independente do modelo de negócio escolhido, as cláusulas contratuais a respeito do fornecimento devem abranger as opções de acesso. Johnson et. al. (2012) esclarecem que isso significa definir se um único utilizador ou vários utilizadores poderão acessar o conteúdo simultaneamente e qual será a forma de autenticação.

Outro aspecto recomendado pelos autores é que estejam previstas contratualmente as possibilidades de rescisão, de reembolso, o período de acordo contratual e cláusulas relativas ao cumprimento das leis dentro da jurisdição legal que rege a biblioteca ou consórcios.

Ainda de acordo com os autores, o arquivamento e direitos pós-término são igualmente objetos de atenção. É preciso considerar previamente o que será mantido de acesso no caso de término ou quebra do contrato. A biblioteca permanecerá com algum tipo de acesso? Haverá possibilidade de obtenção dos metadados? Há uma taxa de manutenção da plataforma mesmo com a aquisição dos arquivos e metadados?

Outra precaução citada por Emery e Stone (2013, p. 17) é o estabelecimento de um limite de preços em contrato. A maioria das bibliotecas não consegue absorver prontamente a taxa padrão de inflação anual de cinco a 12 por cento em média na maioria das assinaturas. Portanto, se um preço subir subitamente de 20 a 30 por cento, por exemplo, o recurso ou conjunto de recursos pode precisar ser cancelado. 


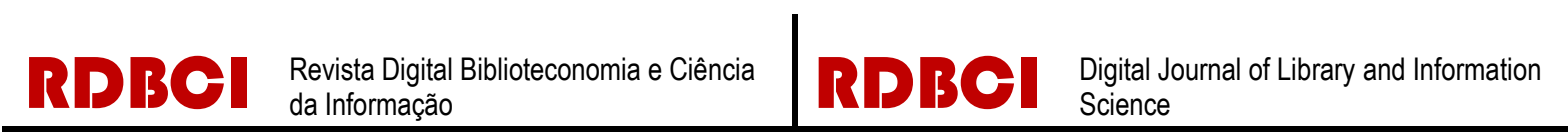

\section{Substituição de Suportes Impressos por Conteúdos Digitais}

Grande parte das unidades de informação tem o acervo formado de itens impressos e eletrônicos. No entanto, muitas vezes o mesmo conteúdo está disponível nos dois suportes. Em função da economia de espaço, recursos financeiros e aperfeiçoamento da distribuição de tarefas da equipe, é preciso decidir sobre a adoção exclusiva do formato eletrônico. Por exemplo, a "desduplicação", que é eliminação de títulos duplicados já adquiridos em vários formatos ocorre muitas vezes na aquisição de coleções de periódicos eletrônicos.

Johnson et. al. (2012) ponderam que, antes de eliminar o equivalente impresso, deve-se analisar:

- Atualidade: recursos eletrônicos com equivalentes em formato impresso não devem ficar para trás dos seus homólogos impressos. Infere-se, portanto, que o recurso eletrônico na maior parte das vezes deve ser o mais atualizado.

- Custo-benefício: o recurso eletrônico deve fornecer um valor acrescentado suficiente sobre o equivalente impresso ou em outros formatos analógicos. $\mathrm{O}$ valor acrescentado pode ser, por exemplo, o aumento da funcionalidade e a maior acessibilidade.

- Precisão e integralidade: o recurso eletrônico deve refletir um conteúdo idêntico ou aumentado em comparação com o equivalente impresso.

- Manutenção de dois formatos: a duplicação de recursos eletrônicos com formatos analógicos equivalentes pode ser considerada se a publicação eletrônica for sujeita a restrições de arquivamento. Se o custo de duplicação é mínimo, a aquisição de vários formatos é a melhor forma de atender às necessidades de diferentes usuários em termos de acesso e comodidade.

\section{Considerações Finais}

Diversos autores brasileiros discorrem sobre a necessidade da elaboração de política de desenvolvimento de coleções em acervos digitais, como Cunha (1999, 2009); Weitzel (2002) e Caribé (2008). Existem ainda estudos específicos que se debruçam sobre o desenvolvimento de coleções digitais, como os de Serra e Silva (2015), Serra (2015), Santanna (2015) e Martins (2016). Há aqueles que se dedicaram a estudar o desenvolvimento de coleções digitais em bibliotecas universitárias, como Dias, Silva e Cervantes (2012), Santana (2013), Magalhães (2014), Silva (2014), Oliveira (2016), Pinheiro (2017) e Santos (2018). 
Embora as unidades de informação façam uso de bases de dados e livros eletrônicos no Brasil há um tempo significativo e a literatura científica brasileira tenha se debruçado sobre o assunto, não foi localizado um estudo nacional que aborde especificamente os assuntos pontuados na revisão de literatura deste artigo.

Por esse motivo, sintetizando as orientações da literatura científica internacional, recomenda-se a observação dos seguintes aspectos relacionados especificamente para coleções digitais:

- Conteúdo: conteúdos adicionais ou funcionalidades de versões digitais de documentos impressos; atualidade e confiabilidade do conteúdo (sobretudo em versões gratuitas) e atualizações frequentes;

- Viabilidade técnica: acesso remoto; autenticação via IP; compatibilidade de hardware, software e navegador;

- Funcionalidade e confiabilidade: mecanismo de busca eficiente, buscas por palavra-chave, pesquisa booleana e no texto integral, possibilidade de percorrer índices e títulos, ordenação por relevância, tesauro, truncamento, histórico de pesquisa e transliteração; possibilidade de exportação para softwares de gestão bibliográfica, envio por correio eletrônico e download sem limitações; folksonomia ou social tagging; metadados interoperáveis; sistema estável com estrutura para receber múltiplos acessos e autenticidade;

- Suporte do fornecedor: treinamento de usuários e oferta de tutoriais; disponibilidade de trials; customização da interface para a identificação da instituição contratante e relatórios estatísticos de acordo com padrões reconhecidos;

- Licenciamento: controle eficiente de acesso com o eventual uso de DRM; definição clara de usuários e locais autorizados;

- Preservação: política de arquivamento, com previsão de responsabilidade da gestão e curadoria digital dos dados; uso de identificadores padronizados;

- Acessibilidade: interface compatível com diretrizes de acessibilidade, acervo apto a ser lido por programas de voz ou ter visualização ampliada; possibilidade de alteração de cor e contraste;

- Usabilidade: interface útil, fácil e agradável; ajudas de navegação; opções de personalização; capacidade de classificação e ordenação de resultados de busca; 
- Aspectos contratuais: estabelecimento explícito da forma de autenticação de usuários, arquivamento e direitos pós-término contratual e limite de reajuste de preço.

Conclui-se que, para o desenvolvimento de coleções digitais eficiente, além dos aspectos acima relacionados também é indicado o estudo dos modelos de negócios praticados, o constante acompanhamento do mercado editorial e conhecimento sobre os dispositivos legais para a compra desses conteúdos, sobretudo em instituições governamentais. Outra recomendação em âmbito acadêmico e profissional é o desenvolvimento de estudos focados nas redes de cooperação, pois acredita-se que essas redes podem expandir ou mesmo potencializar os recursos envolvidos na formação e gestão de coleções digitais.

\section{Referências}

ARORA, Jagdish. Digital Libraries. Gujarat: INFLIBNET Centre, 2018. E-book. Disponível em: http://lisp8.epgpbooks.inflibnet.ac.in/. Acesso em: 02 abr. 2019.

BASTIEN. J.M. Christian. Usability testing: a review of some methodological and technical aspects of the method. International journal of medical informatics. v. 7 9, 2010. Disponível em:

http://www-sciencedirectcom.ez1.periodicos.capes.gov.br/science/article/pii/S1386505608002098. Acesso em: 01 abr. 2019.

BITTAR, Ana Carolina. Digital Rights Management: Concorrência e acesso ao conhecimento no mercado de livros digitais. 2015. 168 f. Dissertação (Mestrado em Direito) Fundação Getúlio Vargas. São Paulo, 2015. Disponível em: http://bibliotecadigital.fgv.br/dspace/handle/10438/13696. Acesso em: 01 abr. 2019.

BOERES, Sonia Araújo de Assis. Política de preservação da informação digital em bibliotecas universitárias brasileiras. 2004. 180 f. Dissertação (Mestrado em Ciência da Informação e Documentação) - Universidade de Brasília, Brasília, 2004.

BUCKLAND, Michael K. The roles of collections and the scope of collection development. Journal of Documentation, 1989, v. 45; n. 3, p. 213-226. Disponível em: https://doi.org/10.1108/eb026844. Acesso em: 30 mar. 2019.

BUCKLAND, Michael K. What will collection developers do? Information Technology and Libraries, v. 14; n. 3, p. 155-159, 1995. Disponível em: https://escholarship.org/uc/item/2v2258mk. Acesso em: 30 mar. 2019.

MELLINS-COHEN, Tasha. Guía amistosa de la versión 5 para bibliotecarios. Tradução: Magaly Bascones. COUNTER: Consistent Credible Comparable. Disponível em: https://www.projectcounter.org/esta-guia-es-una-introduccion-simplificada-al-codigo-depractica-de-counter-version-5-para-bibliotecarios-y-otros-usuarios/friendly-guide-spanish/ Acesso em: 15 ago. 2019. 
CARIBÉ, Rita de Cássia do Vale A aplicação do desenvolvimento e gerenciamento de coleções na construção de repositórios institucionais. Informação \& Sociedade: Estudos, v. 18, n. 2, p. 25-40, 2008. Disponível em: http://www.brapci.inf.br/v/a/5038. Acesso em: 30 mar. 2019.

CORDEIRO, Alexander Magno; OLIVEIRA, Glória Maria de; RENTERÍA, Juan Miguel; GUIMARÃES, Carlos Alberto. Revisão sistemática: uma revisão narrativa. Revista do Colégio Brasileiro de Cirurgiões. Rio de Janeiro, v. 34, n. 6, p. 428-431, dez. 2007. Disponível em: http://www.scielo.br/scielo.php?script=sci_arttext\&pid=S010069912007000600012\&lng=en\&nrm=iso. Acesso em: 02 abr. 2019.

COSTA, Raquel P.; CUNHA, Murilo B. Modelo de negócios de livros eletrônicos para bibliotecas. Informação e Sociedade, João Pessoa, v. 25, n. 3, p. 7-19, set./dez. 2015. Disponível em: http://www.ies.ufpb.br/ojs/index.php/ies/article/view/26945. Acesso em: 02 abr. 2019.

CUNHA, Murilo Bastos da. Desafios na construção de uma biblioteca digital. Ciência da Informação. Brasília, v. 28, n. 3, p. 257-268, set./dez. 1999. Disponível em: http://www.scielo.br/pdf/ci/v28n3/v28n3a3.pdf. Acesso em: 02 abr. 2019.

CUNHA, Murilo Bastos da. Bibliografia sobre o fluxo do documento na biblioteca digital. DataGramaZero: Revista de Ciência da Informação, v. 10, n.5, 2009. Disponível em: http://www.dgz.org.br/out09/Art_01.htm. Acesso em: 02 abr. 2019.

DIAS, Maria Matilde Kronka; PIRES, Daniela. Formação e desenvolvimento de coleções de serviços de informação. São Carlos: EduFSCar, 2003. 71 p. (Série apontamentos).

DIAS, Geneviane Duarte; SILVA, Teresinha Elisabeth da; CERVANTES, Brígida Maria Nogueira. Política de desenvolvimento de coleções para documentos eletrônicos: tendências nacionais e internacionais. Encontros Bibli: Revista Eletrônica de Biblioteconomia e Ciência da Informação, Florianópolis, v. 17, n. 34, 2012, p. 42-56. Disponível em: https://periodicos.ufsc.br/index.php/eb/article/view/1518-2924.2012v17n34p42. Acesso em: 30 mar. 2019.

EMERY, Jill; STONE, Graham. Investigation of new content. In: EMERY, Jill; STONE, Graham. Techniques for Electronic Resource Management. Chicago: Library Technology Reports, $\quad$ n. $2, \quad$ Feb. 2013. 2 Disponível em: https://journals.ala.org/index.php/ltr/article/view/4734. Acesso em: 30 mar. 2019.

FRANKILIN, Brinley; KYRILLIDOU, Martha; PLUM, Terry. From usage to user: library metrics and expectations for the evaluation of digital libraries. In: TSAKONAS, Giannis; PAPATHEODOROU, Christos (Edit.). Evaluation of digital libraries: an insight into useful applications and methods. Oxford: Chandos Pub., 2009. xxiv, 275 p.

GREGORY, Vicki L. Collection development and management for 21st century library collections: an introduction. New York: Neal-Schuman, c2011. xvii, 261 p.

HORAVA, Tony. Challenges and possibilities for collection management in a digital age. Library Resources \& Technical Services. v. 54 n. 3, 2010. Disponível em: http://dx.doi.org/10.5860/lrts.54n3.142. Acesso em: 02 abr. 2019.

\begin{tabular}{|l|l|l|l|l|l|}
\hline (C) RDBCl: Rev. Digit. Bibliotecon. Cienc. Inf. & Campinas, SP & v.17 & $1-20$ & e019025 & 2019 \\
\hline
\end{tabular}


JOHNSON, Sharon; EVENSEN, Ole Gunnar; GELFAND, Glenda Lammers; SIPE, Lynn; ZILPER, Nadia. Key Issues for e-Resource Collection Development: A Guide for Libraries. Haia: IFLA, 2012. Disponível em: https://www.ifla.org/publications/key-issues-for-eresource-collection-development-a-guide-for-libraries. Acesso em 02 abr. 2019.

KAVANAGH, Rosemary; SKÖLD, Beatrice Christensen (ed.). Bibliotecas para cegos na era da informação: diretrizes de desenvolvimento. São Paulo: Imprensa Oficial do Estado de São Paulo, 2009. 99 p. 99 Disponível http://www.santoandre.sp.gov.br/pesquisa/ebooks/343463.pdf. Acesso em: 02 abr. 2019

LIBRARY OF CONGRESS. PDF/A-1, PDF for Long-term Preservation, Use of PDF. Sustainability of Digital Formats: Planning for Library of Congress Collections 1.4. Washington, 2019.2 Disponível em: http://www.loc.gov/preservation/digital/formats/fdd/fdd000125.shtml. Acesso em: 15 ago. 2019.

MAGALHÃES, Carolina de Souza Santana. Seleção de coleções de livros digitais nas universidades públicas brasileiras. 2014. Dissertação (Mestrado em Ciência da Informação) - Universidade Federal da Bahia, Salvador, 2014. Disponível em: https://repositorio.ufba.br/ri/handle/ri/15021. Acesso em: 02 abr. 2019.

MALHEIROS, Tania Milca de Carvalho. Produtos e serviços de informação para pessoas com deficiência visual. 2019. Tese (Doutorado em Ciência da Informação) - Universidade de Brasília, Brasília, 2019. Disponível em: http://repositorio.unb.br/handle/10482/34969. Acesso em: 14 ago. 2019.

MARTINS, Robson Dias. Proposta teórica de criação de plataforma para gerenciamento de e-books. Mestrado (Mestrado em biblioteconomia) - Universidade Federal do Estado do Rio de Janeiro, 2016. Disponível em: https://goo.g1/UkuVn3. Acesso em: 02 abr. 2019.

McNAUGHT, Alistair; HUW, Alexander. Ebooks and accessibility. In: WOODWARD, H. (ed.). Ebooks in Education: Realizing the Vision. London: Ubiquity Press, 2014. p. 35-49. Disponível em:_https://www.ubiquitypress.com/site/books/10.5334/bal/read/. Acesso em: 02 abr. 2019.

NATIONAL INFORMATION STANDARDS ORGANIZATION. A framework of guidance for building good digital collections. 3. ed. Baltimore (MD): NISO, 2007. 100 p. Disponível em: www.niso.org/publications/rp/framework3.pdf. Acesso em: 02 abr. 2019.

NATIONAL INFORMATION STANDARDS ORGANIZATION. Standardized Usage Statistics Harvesting Initiative (SUSHI) Protocol (ANSI/NISO Z39.93-2014). Baltimore (MD): NISO, 2019. Disponível em: https://www.niso.org/standards-committees/sushi. Acesso em: 15 ago. 2019.

NIELSEN, Jacob. Usability 101: Introduction to Usability. In: Nielsen Norman Group. Evidence-Based User Experience Research, Training, and Consulting. 2012. Disponível em:

\begin{tabular}{l|l|l|l|l|l|}
\hline (c) RDBCl: Rev. Digit. Bibliotecon. Cienc. Inf. & Campinas, SP & v.17 & $1-20$ & e019025 & 2019 \\
\hline
\end{tabular}


https://www.nngroup.com/articles/usability-101-introduction-to-usability/. Acesso em: 02 abr. 2019.

OLIVEIRA, Adriana Aparecida de. Avaliação das coleções de livros digitais da Universidade Federal de Juiz de Fora: a perspectiva do usuário. 2016. Dissertação (Mestrado em Biblioteconomia) - Universidade Federal do Estado do Rio de Janeiro, Rio de Janeiro,

2016. Disponível em: http://www.repositoriobc.unirio.br:8080/xmlui/handle/unirio/11139?show=full. Acesso em: 02 abr. 2019.

PINHEIRO, Liliane Vieira. O desenvolvimento de coleções em bibliotecas universitárias na perspectiva dos desafios da pós-modernidade: diretrizes sob o olhar da Teoria da Complexidade e da análise de domínio. 2017. Tese (Doutorado em Ciência da Informação) Universidade Federal de Santa Catarina, 2017. Disponível em: https://goo.gl/EPnBuA. Acesso em: 02 abr. 2019.

RODRIGUES, Eloy; CARVALHO, José. Gestão e Organização da Coleção Digital. Lisboa: Ministério da Educação e Ciência, 2013. Disponível em: http://www.rbe.minedu.pt/np4/file/871/be_rbe_3.pdf. Acesso em: 08 out. 2018.

ROMERO, Juan José Fuentes. Las definiciones de la colección de materiales de las bibliotecas. Los conceptos de desarrollo y gestión de las colecciones: un estado de la cuestión. Boletín de la Asociación Andaluza de Bibliotecarios, n. 88-89, jul. 2007. Disponível em: https://dialnet.unirioja.es/servlet/articulo?codigo=2695898. Acesso em: 02 abr. 2019.

SANTANA, Carolina Souza. Seleção de coleções de livros digitais nas universidades públicas brasileiras. 2013. Dissertação (Mestrado em Ciência da Informação) - Universidade Federal da Bahia, 2013. Disponível em: https://goo.g1/rRdEf. Acesso em: 02 abr. 2019.

SANTANNA, Jorge. Desafios para a gestão de estoques de informação frente às coleções em diferentes contextos. Revista ACB: Biblioteconomia em Santa Catarina, v. 20, n. 3, 2015. Disponível em: https://revista.acbsc.org.br/racb/article/view/1092. Acesso em: 25 abr. 2018.

SANTOS, Ana Rosa dos. Subsídios para a elaboração de políticas de gestão de coleções de livros eletrônicos: Uma proposta para as bibliotecas universitárias federais brasileiras. Rio de Janeiro, 2018. 214 f. Dissertação (Mestrado Profissional em Biblioteconomia) - Universidade Federal do Estado do Rio de Janeiro, Rio de Janeiro, 2018. Disponível em: http://www.repositorio-bc.unirio.br:8080/xmlui/handle/unirio/12356. Acesso em: 25 abr. 2018.

SERRA, Liliana Giusti. Os livros eletrônicos e as bibliotecas. São Paulo, 2015. Dissertação (Mestrado em Ciência da Informação) - Universidade de São Paulo, São Paulo, 2015. Disponível em: http://www.teses.usp.br/teses/disponiveis/27/27151/tde-01122015-101516/ptbr.php. Acesso em: 12 jul. 2018.

SERRA, Liliana Giusti; SILVA, José Fernando Modesto da. Livros digitais em bibliotecas. Encontro Nacional de Pesquisa em Ciência da Informação, v. 16, 2015. Disponível em: http://www.periodicos.ufpb.br/ojs/index.php/itec/article/view/26756.Acesso em: 25 abr. 2018. 
SCHMIDT, Karen. Past perfect, future tense: a survey of issues in collection development. Library Collections, Acquisitions \& Technical Services. v. 28, p. 360-372, 2004.

SILVA, Fabiana Vilar. Subsídios para a elaboração de uma política de depósito mandatória em repositórios institucionais brasileiros: uma análise de estudos de casos a partir de elementos de desenvolvimento de coleções. 2014. Dissertação (Mestrado em Biblioteconomia) - Universidade Federal do Estado do Rio de Janeiro, Rio de Janeiro, 2014. Disponível em: https://goo.gl/2P57mN. Acesso em: 25 abr. 2018.

WEITZEL, Simone. O desenvolvimento de coleções e a organização do conhecimento: suas origens e desafios. Perspectivas em Ciência da Informação, v. 7, n. 1, p. 61-67, 2002. Disponível em: http://www.brapci.inf.br/v/a/3289. Acesso em: 25 abr. 2018.

VERGUEIRO, Waldomiro. Seleção de materiais de informação: princípios e técnicas. 3. ed. Brasília: Briquet de Lemos 2010. 120 p.

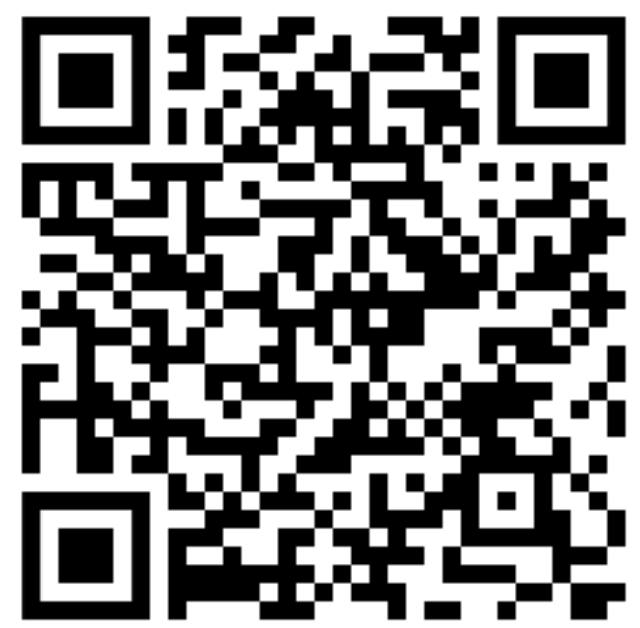

